\section{Cloning Full-Length, Cap-Trapper-Selected cDNAs by Using the Single-Strand Linker Ligation Method}

BioTechniques 30:1250-1254 (June 2001)

\begin{abstract}
We have developed the single-strand linker ligation method (SSLLM), which uses DNA ligase to add a dsDNA linker to singlestranded (ss) full-length cDNA. The linkers have random 6-bp $\left(d N_{6}\right.$ or $\left.d G N_{5}\right) 3^{\prime}$ overhangs that can ligate to any cDNA sequence, thereby facilitating the production of cDNA libraries with titers exceeding $1 \times 10^{6}$ independent clones. We confirmed that the $5^{\prime}$ ends of cDNA inserts cloned by using SSLLM are full-length and include the $5^{\prime}$ untranslated regions. The great advantage of our method is that the elimination of the GC tail simplifies the sequencing and protein translation of the full-length clones. Further, our method tags ss cDNAs more efficiently than does the traditional RNA ligase reaction.
\end{abstract}

\section{INTRODUCTION}

We are constructing the mouse fulllength cDNA encyclopedia, a comprehensive collection of full-length cDNAs (2-4) and their complete sequences. These will contribute to a wide range of biological studies, genetics, diagnostics, and drug development. We have been using G-tailing to prime the synthesis of the second-strand cDNA of Cap-Trapper libraries $(2,3)$, which sometimes affects sequencing and translation. Alternative methods for priming the second-strand cDNA are homopolymeric tailed priming vector (17), RNA ligation (1), which, because of low efficiency, requires PCR $(5,16$, 24) and, worse, sequence preference $(1,7,8,13)$, and RNaseH/DNA polymerase I synthesis, which causes trimming of the $5^{\prime}$ end cDNAs $(6,11)$. We describe the preparation of Cap-Trapper cDNA libraries (2) with the new singlestrand linker ligation method (SSLLM), based on joining ds linkers having a random 6-bp protruding ends to fulllength ss cDNA with DNA ligase.

\section{MATERIALS AND METHODS}

\section{Oligonucleotides and Linkers}

The oligonucleotides (Life Technologies, Tokyo, Japan) were purified as described in Reference 19. We prepared the GN5 linker by mixing oligonucleotides A (5'-AGAGAGAGAG CTCGAGCTCTATTTAGGTGACACTATAGAACCAGNNNNN-3') and B (5'-TGGTTCTATAGTGTCACCTAA ATAGAGCTCGAGCTCTCTCTCT-3'; $5^{\prime}$ end phosphorylated). The $\mathrm{N}_{6}$ linker was prepared by mixing oligonucleotides B and C (5'-AGAGAGAGAGCTCGAGCTCTATTTAGGTGACACTATAGAACCANNNNNN-3'), both in 100 $\mathrm{mM} \mathrm{NaCl}$ and incubated at $65^{\circ} \mathrm{C}$ for 5 $\min , 45^{\circ} \mathrm{C}$ for $5 \mathrm{~min}, 37^{\circ} \mathrm{C}$ for $10 \mathrm{~min}$, and $25^{\circ} \mathrm{C}$ for $10 \mathrm{~min}$. The sequence for testing "check DNA" was 5'-GAG AGAGAGAAGGATCCAAGAGCTCTTTTTTTTTTTTTTTTVN-3' (V = A, $\mathrm{G}$, or $\mathrm{C} ; \mathrm{N}$ represents any nucleotide).

\section{Pilot Testing of SSLLM}

DNA Ligation Kit version 2 (Takara, Tokyo, Japan) was used for SSLLM. Ligation substrates, Solution I, and Solution II were always mixed in the ratio of 1:2:1 and always incubated at $10^{\circ} \mathrm{C}$. For testing, we labeled $2 \mu \mathrm{g}$ check DNA with $3 \mu \mathrm{L}[\alpha-32 \mathrm{P}] \mathrm{ATP}$ $(3000 \mathrm{Ci} / \mathrm{mmol}, 10 \mathrm{mCi} / \mu \mathrm{L}$, Amersham Pharmacia Biotech; Piscataway, NJ, USA) and T4 polynucleotide kinase (Takara), followed by phenol/ chloroform extraction and ethanol precipitation (referred to as "purification") (19). For the SSLLM, we incubated 10 ng $32 \mathrm{P}$-labeled check DNA and $100 \mathrm{ng}$ mixed linkers (molar ratio of $\mathrm{N}_{5}: \mathrm{GN}_{5}$, 1:4) in $20 \mu \mathrm{L}$ for $30 \mathrm{~min}$ or overnight. To assess the efficiency of linker ligation, $2 \mu \mathrm{L}$ samples were run on a $10 \%$ denaturing polyacrylamide gel (19). The image analysis was performed with BAS 2000 bio-imaging system (Fujifilm, Tokyo, Japan).

\section{Using the SSLLM with a Test cDNA}

To determine the ligation conditions for cDNAs, a test first-strand cDNA from $5 \mu \mathrm{g}$ 7.5-kb poly(A)-tailed RNA (Life Technologies) (3) was used, except that Cap-Trapping was omitted with $[\alpha-32 \mathrm{P}] \mathrm{dGTP}$ as a tracer. We combined $50 \mathrm{ng} 7.5-\mathrm{kb}$ cDNA at various amounts (200 ng to $2 \mu \mathrm{g}$ ) of linker $\left(\mathrm{N}_{6}\right.$ or $\left.\mathrm{GN}_{5}\right)$ in $30 \mu \mathrm{L}$ reaction volume and incubated overnight. Samples were incubated with $0.2 \mathrm{mg} / \mathrm{mL}$ proteinase $\mathrm{K}$ in $10 \mathrm{mM}$ EDTA/0.2\% SDS (reaction volume, $40 \mu \mathrm{L}$ ) at $45^{\circ} \mathrm{C}$ for $15 \mathrm{~min}$. After phenol/chloroform extraction and back-extraction (3), linkers in excess were removed with Sephacryl ${ }^{\circledR}-300$ (Amersham Pharmacia Biotech) followed by isopropanol precipitation. To check the efficiency of second-strand cDNA, we used $10 \mathrm{ng}$ purified samples in a $10-\mu \mathrm{L}$ reaction containing $1 \mu \mathrm{L} 10 \times$ ExTaq $^{\text {TM }}$ buffer (Takara), $1 \mu \mathrm{L} 2.5 \mathrm{mM}$ dNTPs, $0.5 \mu \mathrm{L}[\alpha-32 \mathrm{P}] \mathrm{dGTP}$, and 0.5 $\mu \mathrm{L}$ Ex-Taq (Takara), incubated at $65^{\circ} \mathrm{C}$ for $5 \mathrm{~min}, 68^{\circ} \mathrm{C}$ for $30 \mathrm{~min}$, and $72^{\circ} \mathrm{C}$ for $10 \mathrm{~min}$, and then analyzed by alkaline gel electrophoresis.

\section{Preparation and Analysis of a Full-Length cDNA Library}

Cap-Trapper full-length first-strand cDNA was prepared from $10 \mu \mathrm{g}$ mouse liver and brain poly(A)+ mRNA (3) and $200 \mathrm{ng}$ was used for both the Gtailed (control) (3) and for the CapTrapper/SSLLM cDNA, to which we ligated $2 \mu \mathrm{g}$ mixed linkers (molar ratio of $\mathrm{N}_{6}: \mathrm{GN}_{5}, 1: 4$ ) overnight in $30 \mu \mathrm{L}$ final reaction volume (Figure 1A). The second-strand cDNA (Figure 1B) was synthesized by adding $6 \mu \mathrm{L} 10 \times$ ExTaq buffer, $6 \mu \mathrm{L} 2.5 \mathrm{mM}$ dNTPs, and $3 \mu \mathrm{L}$ ExTaq (Takara) in $60 \mu \mathrm{L}$. After $15 \mathrm{~s}$ at $65^{\circ} \mathrm{C}, 3-\mu \mathrm{L}$ aliquots were labeled with $0.5 \mu \mathrm{L}[\alpha-32 \mathrm{P}] \mathrm{dGTP}$ to run on alkaline gel electrophoresis; incubation followed at $65^{\circ} \mathrm{C}$ for $5 \mathrm{~min}, 68^{\circ} \mathrm{C}$ for 30 $\mathrm{min}$, and $72^{\circ} \mathrm{C}$ for $10 \mathrm{~min}$, followed by purification of the samples (19). In control reactions, we used cDNA without linkers. cDNA was digested with B amHI (25 U/ $\mu$ g cDNA; New England Biolabs, Beverly, MA, USA) and XhoI (25 U/ $\mu \mathrm{g}$ cDNA; New England Biolabs) at $37^{\circ} \mathrm{C}$ for $1 \mathrm{~h}$ (Figure $1 \mathrm{C}$ ) and purified (3). We ligated $10 \mathrm{ng}$ cDNA to $190 \mathrm{ng}$ of the lambda FLC-I vector, which carries two loxP sites (12) (P. Carninci, manuscript in preparation). 


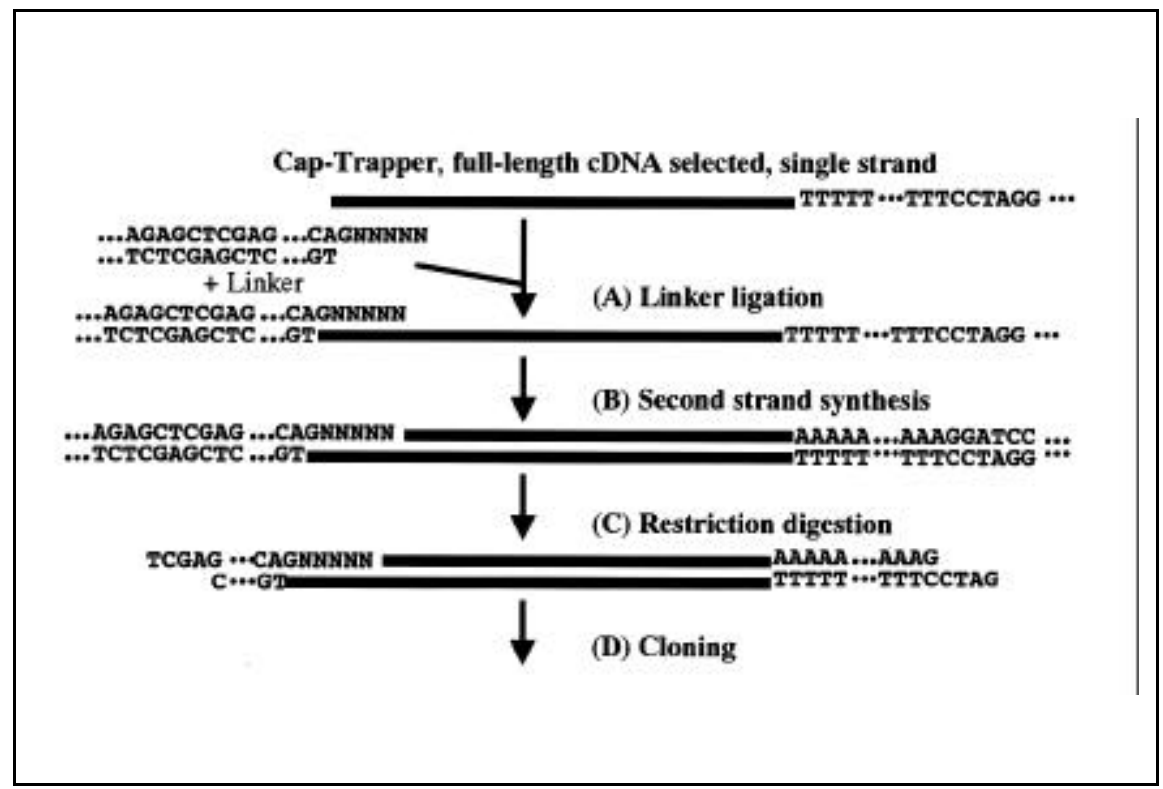

Figure 1. Overview of the preparation of a Cap-Trapper/SSLLM full-length cDNA library. Cap-Trapper full-length cDNA ligation with the linkers (GN in this example). (A) The protruding part is N instead of GN5 for No linkers; (B) second-strand cDNA synthesis; (C) restriction digestion; and (D) cloning.

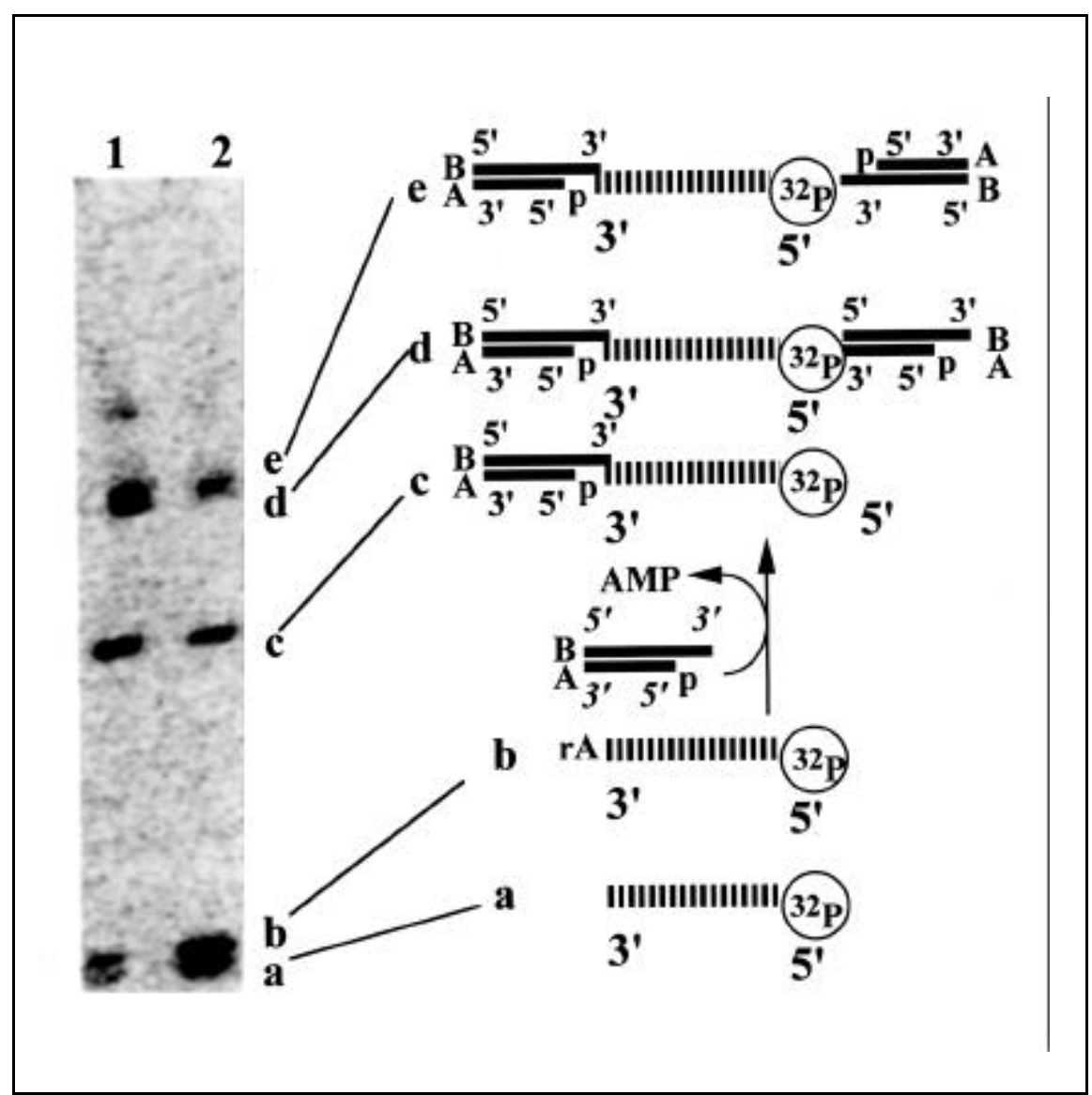

Figure 2. Pilot study of SSLLM check DNA analysis with polyacrylamide gel. Ligation incubations were performed overnight (lane 1) or for $30 \mathrm{~min}$ (lane 2). Bands a-e are indicated in the right column. Band a, unligated check DNA; band c, main ligation product; bands d and e, putative concatamers. Band b, ADP/check DNA intermediate (14). 


\section{Short Technical Reports}

After packaging (MaxPlax ${ }^{\mathrm{TM}}$; Epicentre, Madison, WI, USA), cDNA libraries were amplified as described (19) in C600 cells. We isolated library phage DNA from $1 \mathrm{~mL}$ amplified phage solution using Wizard ${ }^{\circledR}$ Lambda Preps DNA Purification System (Promega, Madison, WI, USA). We converted one-fourth of the phage DNA to plasmid with $1 \mathrm{U}$ cre-recombinase at $37^{\circ} \mathrm{C}$ for $1 \mathrm{~h}$ in $300 \mu \mathrm{L}$ as recommended (Novagen, Madison, WI, USA), and then purified (19). The bulk-excised plasmid libraries were electroporated into $\mathrm{DH} 10 \mathrm{~B}^{\mathrm{TM}}$ cells (Life Technologies). We extracted the plasmids (19) from 36 clones/library and sequenced the inserts from the $5^{\prime}$ ends with M13 forward primer, BigDye ${ }^{\mathrm{TM}}$ Terminators (Applied Biosystems, Foster City, CA, USA), using an ABI PRISM ${ }^{\mathrm{TM}} 3700$ DNA sequencer (Applied Biosystems). We searched with BLAST2, a nonredundant database including GenBank $^{\circledR}$, EMBL, DDBJ, and PDB sequences, but not EST, STS, GSS, or HTGS sequences.

\section{RESULTS AND DISCUSSION}

\section{Designing the Linkers for SSLLM}

Figure 1 outlines the ligation steps for preparing Cap-Trapper/SSLLM full-length cDNA libraries. Linkers had 3 ' overhangs of six degenerate bases to efficiently anneal the ss cDNAs while keeping the complexity of the annealing oligonucleotide low (4096 combinations) and, thus, hybridize efficiently. Moloney murine leukemia virus (MMLV) reverse transcriptase adds, either in a template-independent or capdependent fashion, 1-3 deoxycytidines to the $3^{\prime}$ terminus of cDNA (20), as confirmed in 112 of 142 clones from an in-house collection of abundantly expressed genes. Therefore, we prepared the $\mathrm{GN}_{5}$ linker (GNNNNN overhang) in addition to the $\mathrm{N}_{6}$ linker (NNNNNN overhang).

\section{Pilot Test of the SSLLM}

We ligated $\mathrm{N}_{6} / \mathrm{GN}_{5}$ (1:4) mixed linkers to the ss check DNA using DNA Ligation Kit version 2, which contains polyethylene glycol (PEG) to acceler- ate the reaction. The efficiency of the check DNA-linker overnight ligation was $88 \%$ (Figure 2, lane 1), much higher than conventional ligation $(10 \%$; data not shown). This use of check DNA was suboptimal because its $3^{\prime}$ (...TTTTVN) is not random, as expected for a population of cDNAs. In fact, only 12 specific linkers were used $(12 / 4096$ fraction or $0.29 \%)$, which are complementary to the $3^{\prime}$ end of the check DNA among the existing $\mathrm{d} / \mathrm{f}$ combinations. For random cDNAs, even with the high GC content of $5^{\prime}$ untranslated regions (UTRs), we do not expect sequence bias because we used a several hundred times excess of linkers. Ligation of check DNA with linkers formed concatamers (Figure 2, lane 1, bands $\mathrm{d}$ and e), which may represent ligation of multiple molecules and is not produced with cDNA because the $5^{\prime}$ ends are not phosphorylated.

\section{Using the SSLLM with a Test cDNA}

We tested the ligation with various amounts of $\mathrm{N}_{6}$ and $\mathrm{GN}_{5}$ linkers and 50 ng of a single 7.5-kb cDNA. When sufficient linker ( $2 \mu \mathrm{g}$ in this example) was used, the second-strand synthesis is efficient and yields products of the same size as the first-strand cDNA (Figure $3 \mathrm{~A}$, lane 8). Since first- and the secondstrand cDNAs have the same electrophoretic profile, there is no detectable internal priming. In contrast, the control lane (Figure 3A, lane 7), which does not contain linker, produced a $15-\mathrm{kb}$ band, which probably re-

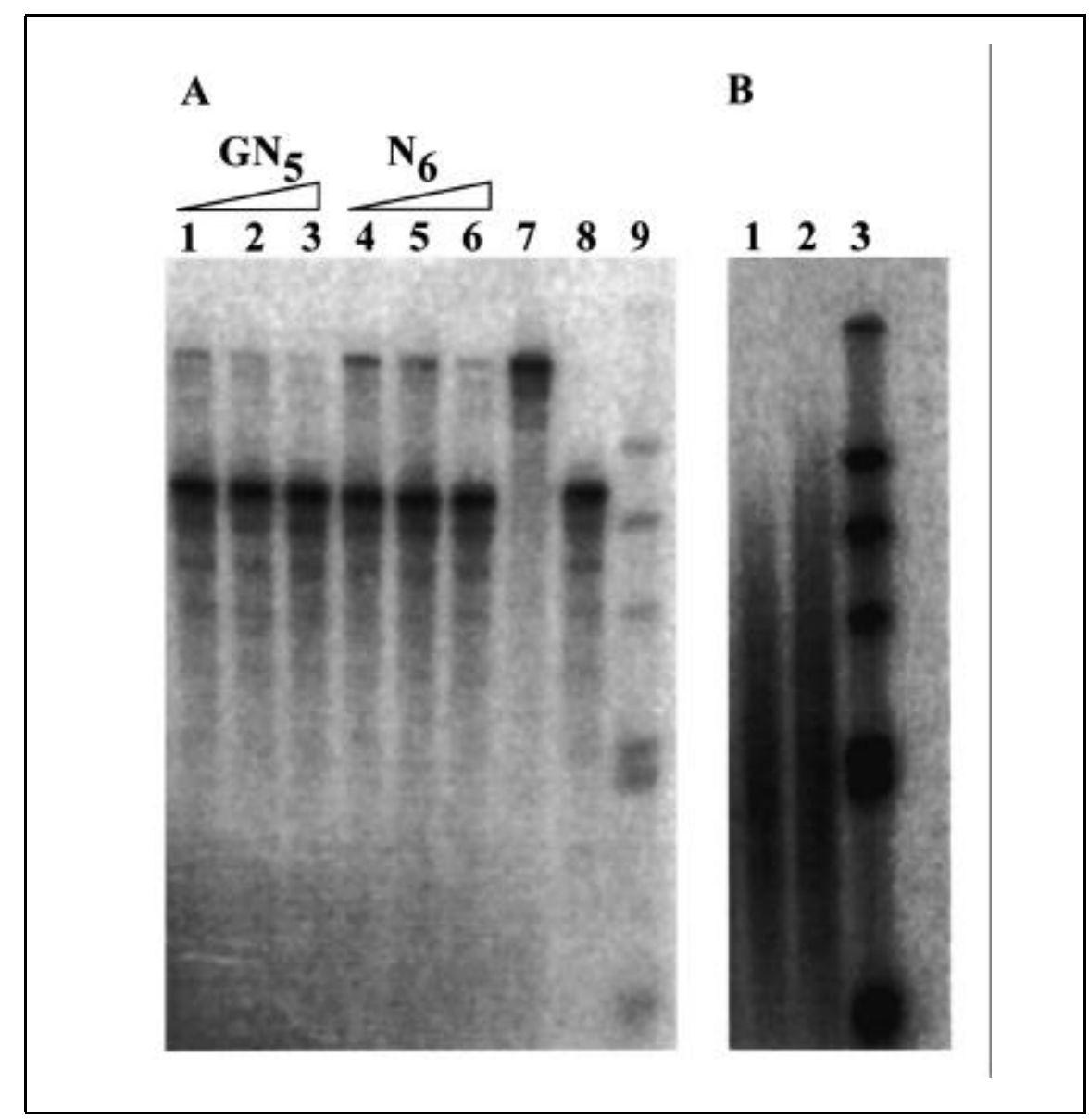

Figure 3. Estimating concentration of linker. (A) Alkaline gel electrophoresis of SSLLM secondstrand cDNA with the GN (lanes 1-3) and $\mathrm{f}$ linkers (lanes 4-6) ligated to the 7.5-kb test cDNA. Quantity of linker: $200 \mathrm{ng}$, lanes 1 and $4 ; 500 \mathrm{ng}$, lanes 2 and 5; $2 \mu \mathrm{g}$, lanes 3 and 6 . Control, linker-free cDNA used as a template (lane 7). Lane 8, first-strand cDNA. Lane 9, HindIII size markers. (B) Size distribution determined by alkaline gel electrophoresis of the first and the second cDNAs from a human mRNA cell line (GCT44): cDNA (lane 1) by Cap-Trap/SSLLM and second-strand cDNA (lane 2). Lane 3, HindIII size markers. 
sults from a hairpin loop at the $3^{\prime}$ end of the first-strand cDNA, which primes the second-strand cDNA $(17,22)$, similar to the pattern obtained with an insufficient quantity of linker. The GN5 linker ligates better than the $\mathrm{N}_{6}$ linker (Figure 3A, lanes 1 and 4) because of the addition of $\mathrm{dC}$ residues by the reverse transcriptase at the $3^{\prime}$ ends of $\mathrm{cD}$ NAs. Although this activity is slightly more effective for the capped mRNAs (20), our data for this and the other three templates (data not shown) suggest that cDNA deriving from uncapped RNA (4) can be efficiently cloned with the GN 5 linker. To maximize the linker-ligation efficiency, we mixed the $\mathrm{GN}_{5}$ and $\mathrm{N}_{6}$ linkers at a 4:1 ratio (not shown).

\section{Preparation and Analysis of a cDNA Library}

We prepared mouse liver and brain cDNA libraries with the Cap-Trapper/G-tailing method (2) and by CapTrapper/SSLLM. With brain cDNAs, $5.83 \mu \mathrm{g}$ Cap-Trapper/G-tailed cDNA and 166 ng Cap-Trapper/SSLLM produced $3.04 \mu \mathrm{g}(52 \%)$ and $73 \mathrm{ng}(44 \%)$ second-strand cDNA, respectively. Since the final phage titers were around $1 \times 10^{6}$ plaques when $10 \mathrm{ng}$ cDNA were used for cloning, this showed that SSLLM is suitable for high yield and titer. Together with the demonstration that there is no shortening of secondstrand cDNA because of internal prim ing (Figure 3B), we think that the cDNA molecules are correctly ligated. Gel filtration with Sephacryl S300 was found essential to eliminate most of the unreacted linkers, which would compete with cDNA and affect the restriction/cloning steps (Figure 1, C and D).

After bulk excision, we sequenced the $5^{\prime}$ ends of clones prepared with Cap-Trapper/G-tailing and Cap-Trapper/SSLLM. Sequence comparison showed that all of the identified clones $(10,16)$ had the first ATG (not shown) and were considered full-length (21), showing the suitability of SSLLM for full-length cDNA cloning.

\section{Advantages of the SSLLM}

Any homopolymeric tail-based cloning is not recommended because of uncontrollable tail length (up to 20000 nucleotides for dA and dT) (14) and sequencing impairment due to slippage, which is not observed with $5^{\prime}$ end direct sequencing of SSLLM clones even with direct colony PCR. This is beneficial to overcome low template concentration and limited plasmid growth due to length or toxicity of cDNA inserts (23). Although the G-tailing reaction is self-limiting to 15-30 nucleotides (14), this has sometimes interfered with sequencing. Long $\mathrm{G}$ stretches, now replaced by SSLLM, may also interact with mammalian $5^{\prime}$ UTRs, for which the average GC content is $60 \%$ (18), forming strong secondary structures in full-length libraries.

Since the annealing efficiency of AT-rich and GC-rich overhangs is equivalent (9), SSLLM linkers should allow the preparation of high-complexity libraries without the sequence bias that was shown for RNA ligase (DNAand RNA-dependent) (7). SSLLM clones are also transcription and translation "friendly" and are suitable for expression cloning $(10,15)$ (not shown). SSLLM also avoids the use of $\mathrm{MnCl}_{2}$ or $\mathrm{CoCl}_{2}$, required in tailing reactions, which have sometimes caused degradation of cDNA. Finally, the SSLLM might be used as universal ssDNA priming method [e.g., in cloning $5^{\prime}$ ends of genes by using rapid amplification of cDNA ends (RACE)], replacing RNA ligase (with DNA substrates) and homopolymer tailing.

\section{REFERENCES}

\footnotetext{
1.Brennan, C.A., A.E. Manthey, and R.I. Gumport. 1983. Using T4 RNA ligase with DNA substrates. Methods Enzymol. 100:3852.

2.Carninci, P., C. Kvam, A. Kitamura, T. Ohsumi, Y. Okazaki, M. Itoh, M. Kamiya, K. Shibata et al. 1996. High-efficiency fulllength cDNA cloning by biotinylated CAP trapper. Genomics 37:327-336.

3.Carninci, P. and Y. Hayashizaki. 1999. High-efficiency full-length cDNA cloning. Methods Enzymol. 303:19-44.

4.Carninci, P., Y. Nishiyama, A. Westover, M. Itoh, S. Nagaoka, N. Sasaki, Y. Okazaki, M. Muramatsu, and Y. Hayashizaki. 1998. Thermostabilization and thermoactivation of thermolabile enzymes by trehalose and its application for the synthesis of full length cDNA. Proc. Natl. Acad. Sci. USA 95:520524.

5.Dumas-Milne-Edwards, J.B., O. Valde-
} 
